\title{
A CASE OF RETROSTERNAL DIAPHRAGMATIC HERNIA
}

BY

\section{R. WARWICK-BROWN}

From the Department of Anatomy, the Medical School, St. Bartholomew's Hospital, London

(RECEIVED FOR PUBLICATION SEPTEMBER 3, 1952)

Following the report of an example of bilateral parasternal diaphragmatic hernia (Warwick-Brown, 1952) an example of a type of hernia in this region is now described from a specimen encountered during a recent routine necropsy at St. Bartholomew's Hospital. This type of hernia has not been described before.

The patient was a woman aged 70 who died in the post-operative period following the abdominoperineal excision of a carcinomatous rectum. The hernia was noted at operation, but had not been previously suspected.
A triple diaphragmatic defect was present, comprising (a) a central gap between the two sternal slips of origin, $(b)$ a dextro-lateral gap between the right sternal and the adjacent costal slips of the muscle, and (c) a sinistro-lateral gap situated between the left sternal and the left costal origins of the muscle (Fig. 1). These three diaphragmatic apertures were of equal extent.

The central diaphragmatic defect gave passage to the peritoneal sac, while the two lateral defects each admitted a mass of extra-peritoneal fat. Each mass of fat was characterized by a peritoneal

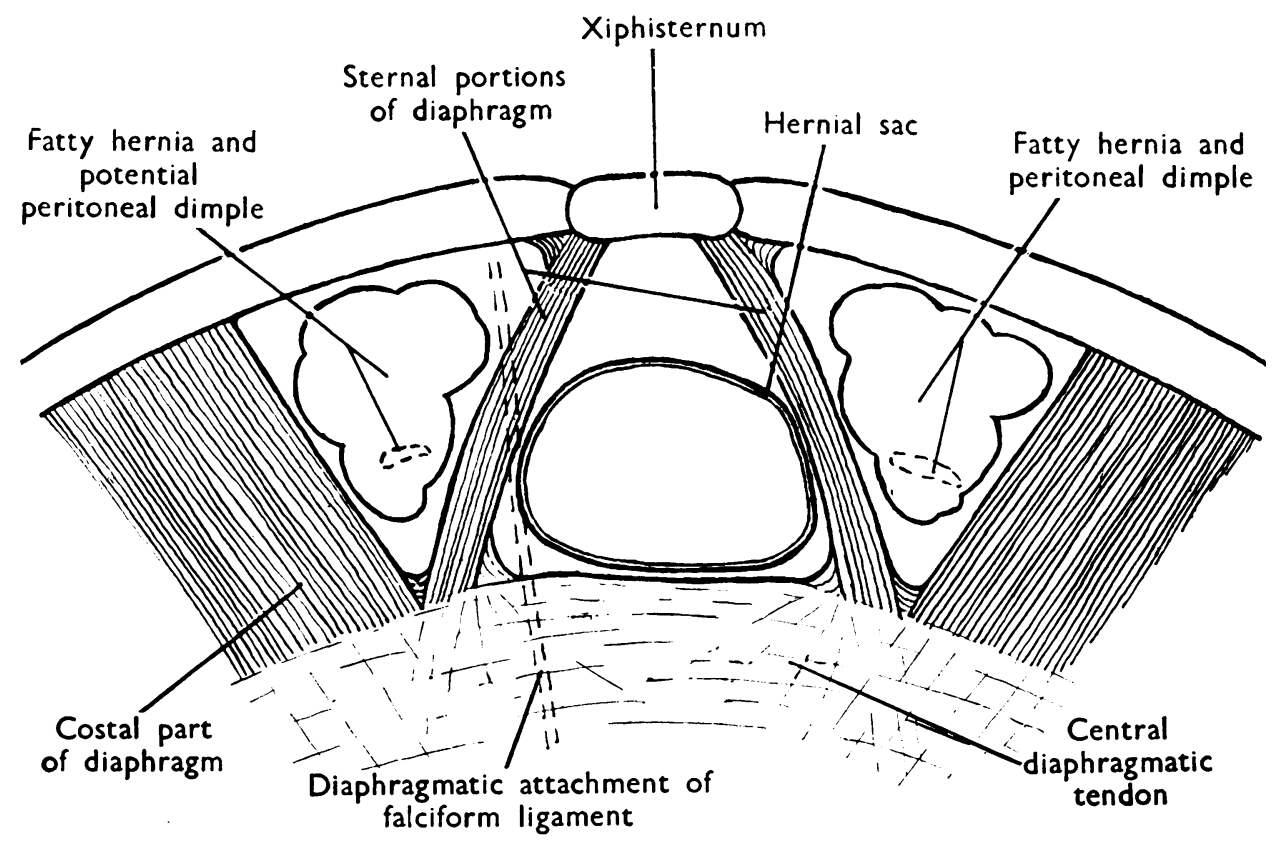

FIG. 1.-The diaphragm from below.

The Hernia

The hernia consisted of a complete peritoneal sac, with attendant extra-peritoneal fat, which had passed through a central diaphragmatic defect situated immediately behind the xiphisternum and so into the anterior mediastinum. "dimple" on its abdominal aspect, suggestive of the potential formation of a parasternal hernial sac.

The oval neck of the peritoneal sac measured $4 \mathrm{~cm} . \times 2 \mathrm{~cm}$., its long axis lying transversely. The sac was complete and irregular in outline, and it 
extended some $10 \mathrm{~cm}$. upwards and to the right, where it invaginated the right mediastinal pleura (Fig. 2). The upper, diaphragmatic attachment of the falciform ligament passed across the right margin of the orifice, but did not enter the sac.

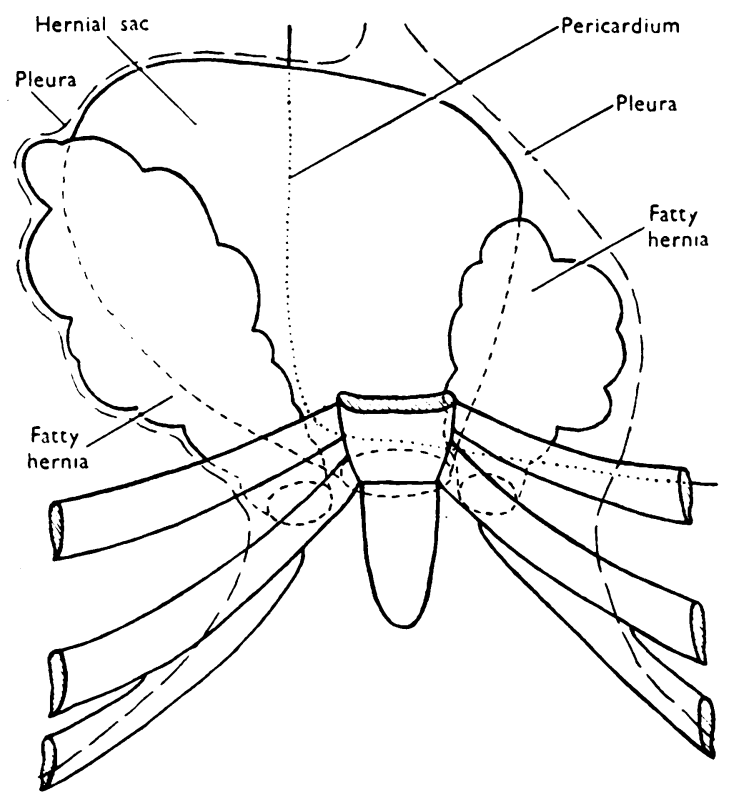

FIG. 2.-Diagram showing the relations of the herniae.

The fatty herniae passed through the lateral diaphragmatic defects on each side, that is, through the enlarged spaces of Larrey. In each case the mass of extruded fat lay antero-lateral to the medianly disposed peritoneal sac, that on the left indenting the extra-pericardial fat and that on the right invaginating the right pleura.

The hernial sac was reported to be empty both at operation and at the subsequent necropsy.

\section{Discussion}

The literature on the subject of parasternal hernia was briefly reviewed in the earlier article
(Warwick-Brown, 1952). All the previously reported diaphragmatic herniae in this region were found to have passed through the space of Larrey on one or other side, that is, to have been truly parasternal in nature. In the present case extraperitoneal fatty herniae occupied these lateral sites, while a peritoneal sac emerged into the thorax between the sternal slips of origin of the diaphragm.

As indicated in the previous article, it would appear that enlargements of the normal spaces of Larrey are congenital defects due to abnormal development of that portion of the diaphragm resulting from fusion of the embryonal septum transversum and body wall components. To this same category must be referred the present specimen, in which, as well as the congenital enlargement of the spaces of Larrey, there exists also a congenital hiatus between the two sternal slips of origin of the diaphragm.

Herniae through abnormally, i.e., congenitally, enlarged spaces of Larrey are properly designated parasternal, inasmuch as their site of exit is placed lateral to the sternum. The secondary intrusion of such parasternal herniae into the retrosternal region does not warrant their being termed retrosternal, notwithstanding the occasional misuse of this term in the literature. The present example illustrates true retrosternal hernia, for the site of exit of the hernial sac is behind, and not lateral to, the sternum. It is suggested that the term "retrosternal hernia " be properly restricted to herniae of this nature.

The specimen briefly described here is preserved in the Pathology Museum of St. Bartholomew's Hospital Medical College, London.

My thanks are due to Professor J. W. S. Blacklock and Dr. R. J. R. Cureton, of the Pathology Department of St. Bartholomew's Hospital, for permission to publish this report, and to Professor A. J. E. Cave for assistance in its preparation.

REFERENCE

Warwick-Brown, R. (1952). Thorax, 7, 266 